\title{
Patterned Planar array Immunosensor For Multianalyte Detection
}

\author{
Randy M. Wadkins, Joel P. Golden, and Frances S. Ligler \\ Naval Research Laboratory, Center for Bio/Molecular Science and Engineering, Code 6900, \\ Washington, DC 20375-5348 \\ (Paper JBO-097 received June 13, 1996; revised manuscript received Oct. 7, 1996; accepted for publication Oct. 25, 1996.)
}

\begin{abstract}
A fluorescence-based immunosensor using a charge-coupled device (CCD) as a detector has been developed for detecting multiple analytes within small (150 $\mu$ l) sample volumes. Wells approximately $2 \mathrm{~mm}$ in diameter were patterned onto glass coverslips using a photoactivated optical adhesive. In a model detection system, four different antigens were covalently attached to the bottoms of these wells, and the polymer subsequently removed to form the sensing surface. The coverslips were mounted over a scientific grade CCD operating at ambient temperature in inverted (multipin phasing) mode. A two-dimensional graded index of refraction (GRIN) lens array was used to focus the sensing surface onto the CCD. Solutions of fluorescently labeled antibodies were then placed on the coverslip, and the amount of antibody bound at each location on the coverslip was determined by quantitative image analysis. Concentrations as low as $50 \mathrm{ng} / \mathrm{ml}$ of Cy5-labeled antibodies could be detected using the sensor. The small footprint and minimal system requirements of the design should facilitate its incorporation into portable biosensors. () 1997 Society of Photo-Optical Instrumentation Engineers.
\end{abstract}

Keywords biosensor; multianalyte; immunoassay; charge-coupled device (CCD); fluorescence; array.

\section{INTRODUCTION}

Biosensors for detection of a variety of analytes have become a topic of considerable interest in recent years. ${ }^{1-4}$ In general, these biosensors are fashioned around immobilization of antibodies for a specific analyte onto a surface. Subsequently, the detection of the bound analyte is measured through transduction of an alteration in the physical properties of the surface (e.g., surface plasmon resonance, electrochemical detection) or through a change in the spectroscopic properties of the system that occurs upon binding of the analyte or a secondary antibody. This latter method of optical detection may utilize absorbance or Raman spectroscopy, or fluorescence from either the bound analyte or a fluorescently labeled secondary antibody. Optical biosensor designs may incorporate planar waveguides as a means of carrying light to the surface, or may use optical fibers. ${ }^{1-6}$

Development of the next generation of biosensors has been focused on the need to detect multiple analytes within a given sample. The use of multianalyte biosensors is a desirable goal from many practical perspectives, since conceivably they would reduce the amount of sample needed for measurement, as well as the time required to probe for multiple analytes. Recent designs of array-type multianalyte biosensors have used multiple antibodies immobilized onto ion-sensitive field-effect

Address all correspondence to Frances S. Ligler. E-mail: fligler@cbmse.nrl.navy.mil transistors $^{7,8}$ or on the ends of optical fiber bundles. ${ }^{9}$

By far, the most widely used technique for biosensor array design is the use of fluorescently labeled probes to quantitate the amount or kind of analyte present. Schemes employing fluorescence have been used in immobilization of peptides and DNA directly to the surface of a charge coupled device (CCD), with excitation of the bound fluorophores accomplished with a fluorescence microscope ${ }^{10,11}$ Similar immobilizations onto CCD chips have also been incorporated into designs using ${ }^{32} \mathrm{P}$ detection of DNA hybridization. Other similar designs utilize arrays of DNA or peptides prepared on glass substrates, which are then subsequently probed with fluorescence microscopes. ${ }^{12-15}$

In the case of fluorescence-based immunosensors, several approaches have been used to generate arrays of antibodies for detection of multiple analytes. Usually, antibodies are deposited in separate wells in or on a waveguide, and detection of the fluorescent analyte is performed using scanning photomultiplier tubes ${ }^{16,17}$ or cooled CCD camera systems. ${ }^{4,18}$

These earlier immunosensor systems, although useful, have required relatively high concentrations of analytes, moving parts, and/or cooled CCD cameras and electronics. Our goal has been to design a multianalyte immunosensor that is easily integrated into portable biosensor devices. Two recent advances have provided the means of combining 


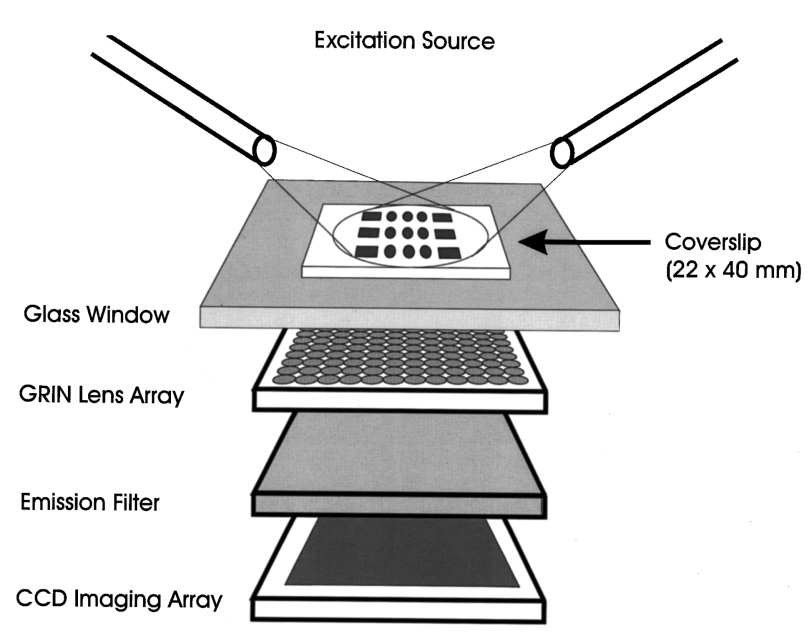

Fig. 1 Schematic diagram of the biosensor. A patterned glass coverslip with covalently bound antibodies is placed on a glass plate mounted on a steel support and illuminated by $635 \mathrm{~nm}$ light from a halogen lamp. The resulting fluorescence from bound antigens is focused onto a scientific grade CCD through a GRIN lens array $(14 \times 31)$ and a 665 -nm long-pass filter. The image formed on the CCD is collected with external electronics.

disposable, patterned sensing surfaces with CCD sensor detection: (1) the development of highly fluorescent, near-IR antibody labeling reagents ${ }^{19,20}$ that emit at wavelengths of maximum quantum yield for scientific grade CCDs; and (2) CCDs that may operate in multipin phasing (MPP) mode, reducing dark current noise at room temperature so that cooling may not be necessary. ${ }^{21}$ In this report, we describe the coupling of these two technologies to produce a compact, array-based immunosensor for detection and quantification of multiple analytes at concentrations as low as $50 \mathrm{ng} / \mathrm{ml}$.

\section{MATERIALS AND METHODS}

\subsection{IMAGING SYSTEM}

The basic design of the biosensor is shown in Figure 1. A scientific grade CCD image sensor (CCD05-30 from EEV, Elmsford, New York) consisting of $1242(\mathrm{H}) \times 1152(\mathrm{~V})$ pixels was used as the detection system. This CCD was operated in inverted (MPP) mode to suppress dark current noise. CCD detectors operated in this mode acquire dark current noise up to 20-fold less quickly than nonMPP CCDs, and improve charge transfer efficiency. The combined effect is that for relatively short exposures these CCDs can be operated at room temperature with significant sensitivity. Half of the vertical pixels were used as a frame store for continuous image acquisition, so each image frame consisted of $1242(\mathrm{H}) \times 576(\mathrm{~V})$ pixels. The readout rate (pixels/s) was maintained at $50 \mathrm{kHz}$ so that each image took $14.8 \mathrm{~s}$ to acquire.

The glass-covered CCD chip was inserted into an appropriate headboard and mounted underneath a metal stage. The video signal output from the chip and electrical power was controlled by a drive circuitry unit (EEV), external to the stage area. The video output signal was collected using a Lab-LC A/D board (National Instruments, Austin, Texas) mounted in a Macintosh LC475 computer, and a program written in THINK C using the A/D board software libraries. Each acquired frame was saved as a 12-bit digital image.

The stage area above the CCD was constructed from a $0.6-\mathrm{cm}$ thick rectangular aluminum plate $21 \times 26 \mathrm{~cm}$. This plate was mounted on four $15.2-\mathrm{cm}$ screws (one at each corner of the plate) and secured to an optical breadboard. A rectangular area 10.1 $\times 8.3 \mathrm{~cm}$ was removed from the aluminum plate near its center. A $0.2-\mathrm{cm}$ wide and $0.2-\mathrm{cm}$ deep ledge was cut into the aluminum plate around the rectangular cutout so that a large glass microscope slide $(10.2 \times 8.3 \times 0.2 \mathrm{~cm}$; Thomas Scientific) just fit into the cutout and was flush with the top of the aluminum plate. The bottom of the glass plate was masked with black spray paint, except for a 3.5$\times 7.3 \mathrm{~cm}$ region in the center. The glass cover slips with immobilized antigens (see later discussion) were placed over the unpainted region for interrogation.

A graded index of refraction (GRIN) lens array was placed beneath the glass plate to focus the surface of the cover slips onto the CCD. Each cylindrical GRIN lens was $0.7 \mathrm{~cm}$ long, with a diameter of $0.09 \mathrm{~cm}$ and a focal length of $0.38 \mathrm{~cm}$ from either end of the lens cylinder. The two-dimensional GRIN lens array was constructed from linear rows of GRIN lenses (Nippon Sheet Glass, Somerset, New Jersey) so that it covered the unmasked area under the glass plate. The array was mounted 3 $\mathrm{mm}$ below the glass plate and $3 \mathrm{~mm}$ above the CCD surface, with the heights adjusted to achieve exact focusing.

The coverslips were interrogated by projecting two beams of light from a halogen projector lamp equipped with two fiber optic bundles (DolanJenner Industries, Lawrence, Massachusetts) through two $635 \pm 10 \mathrm{~nm}$ bandpass filters (Omega Optical, Brattleboro, Vermont) and onto the coverslip surface. Light was projected at a 45-deg angle from the coverslip surface, and was introduced from two sides to improve lighting homogeneity. A $5 \times 5$-cm square, 665-nm long-pass filter (Schott Glass, Duryea, Pennsylvania) was placed between the GRIN lens array and the CCD to reduce detection of excitation light.

\subsection{CONSTRUCTION OF ANTIBODY ARRAYS}

Antibody arrays were formed on glass coverslips $(22 \times 40$ mm, No. 1.5; VWR Scientific, Media, Pennsylvania) by generating a series of wells using the photomask shown in Figure 2. The mask was constructed by printing the pattern onto standard overhead projector transparencies. This mask consisted 


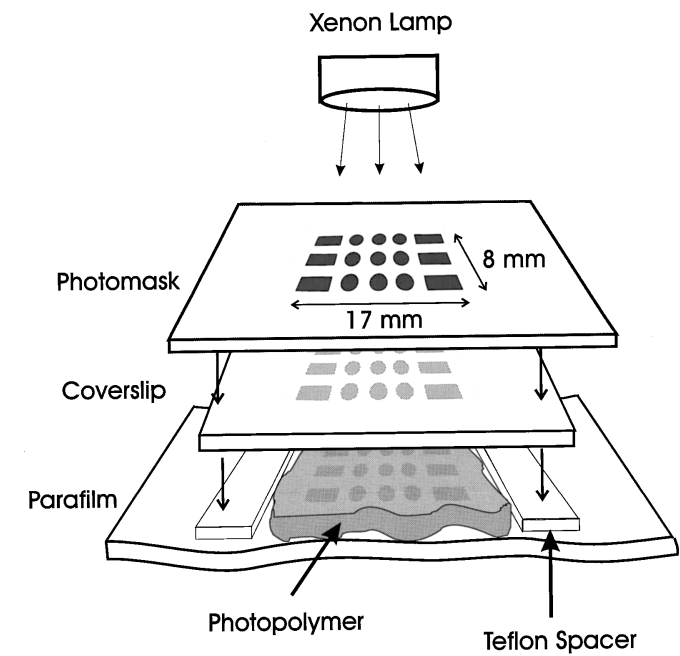

Fig. 2 Construction of the antibody pattern. A photopolymerizable polymer is deposited between a glass coverslip (supported by Teflon spacers) and a piece of laboratory parafilm. The indicated pattern was printed onto standard overhead transparency material and placed above the glass slide. Illumination with a xenon lamp resulted in polymerization of the polymer everywhere but within the dark areas of the mask. The unpolymerized material was removed with an acetone-soaked cotton ball. Immunoglobulins were then covalently attached to the exposed, uncovered glass.

of 6 rectangular regions that served as control wells for lighting variations and 9 circular regions.

Wells were formed by placing a $0.2 \mathrm{~mm}$ layer of Norland Optical adhesive No. 63 (Norland Products, Brunswick, New Jersey) between the glass coverslip and a layer of laboratory parafilm. The pattern was then placed over the glass and unfiltered light from a 100-W xenon lamp was directed onto the pattern for $2 \mathrm{~min}$, as per the manufacturer's directions for precuring. The pattern and parafilm were then removed to expose the moderately polymerized adhesive. An acetone-soaked cotton swab was used to remove unpolymerized adhesive from the wells. This left polymer-free wells with glass bottoms within the polymerized coating. The cleaned coverslips with the polymers were placed under a 360-nm UV lamp (Fisher Scientific) for 30 min. The fully cured polymer on glass was stored overnight in the dark.

The polymer-coated glass coverslips were then silanized to activate the wells for crosslinking to antibodies. ${ }^{22}$ The coverslips were coated with a $4 \%$ solution of 3-mercaptopropyltrimethoxysilane (Fluka) in toluene. The silanized coverslips were then incubated with $2 \mathrm{mM} \quad \gamma$-maleimidylbutyryl succinimide (Fluka) in ethanol for $1 \mathrm{~h}$ prior to the addition of antigen.

The following immunoglobulins (Ig) were purchased from Jackson ImmunoResearch (West Grove, Pennsylvania): horse immunoglobulin G (IgG), Cy5-labeled rabbit anti-horse, goat IgG, Cy5labeled rabbit anti-goat, chicken IgG (IgY), Cy5labeled rabbit anti-chicken, mouse IgG, and Cy5- labeled rabbit anti-mouse IgG. A $0.25 \mathrm{mg} / \mathrm{ml}$ solution of each IgG in phosphate buffered saline (PBS) was pipetted into separate wells. The horse IgG was placed into the rectangular wells to serve as the control sample. The goat, chicken, and mouse IgG's were placed in the three wells in each row, so that each measurement could be made in triplicate. After incubating with these antibody solutions for $1 \mathrm{~h}$, the coverslips were rinsed with distilled water and stored in $1.5 \%$ bovine serum albumin (BSA) in PBS at $4{ }^{\circ} \mathrm{C}$.

\subsection{IMMUNOASSAYS}

Direct immunoassays were performed using Cy5labeled rabbit anti-species IgG diluted in PBS/ $0.05 \%$ Tween-20 detergent (to reduce nonspecific binding of antibodies) to the desired concentrations. The polymer coating that formed the wells during antigen deposition was easily removed from the coverslips after the coverslips were soaked overnight in BSA-PBS. These exposed coverslips, with antigens immobilized at specific locations, were immersed in $1.5 \%$ BSA for 20 min to block newly exposed, nonspecific binding locations on the glass surface.

After BSA blocking, the coverslips were rinsed with distilled water and air dried. A PAP Pen (wax in toluene; The Binding Site Inc., San Diego, California) was used to encircle the assay area to reduce the volume of sample required for the experiment. The coverslips were then secured over the transparent area of the glass plate in the imaging platform.

Images of the coverslips in PBS buffer solutions were taken as background images to be subtracted from the immunoassay images. A $150 \mu \mathrm{l}$ aliquot of $500 \mathrm{ng} / \mathrm{ml}$ Cy5-rabbit anti-horse IgG was initially applied to the coverslip. After $15 \mathrm{~min}$, this solution was rinsed off, the slide illuminated, and an image captured to visualize the rectangular areas on the coverslip. The variation in fluorescence between these rectangular areas accounted for lighting nonuniformity within the image. For increasing concentrations of Cy5-labeled rabbit anti-species IgG, a $150 \mu \mathrm{l}$ aliquot of each solution was placed on top of the coverslip and allowed to incubate for $15 \mathrm{~min}$. The slide was then rinsed twice with PBS- $0.05 \%$ Tween 20, and the coverslip was illuminated with the halogen lamp. The fluorescence from the bound Cy5-labeled antibody was then visualized by capturing the image. Higher concentrations were added sequentially to generate a titration curve. Species were added in the order: rabbit anti-goat IgG, rabbit anti-chicken IgG, and rabbit anti-mouse IgG.

The amount of antibody captured was quantitated from the CCD images using NIH Image (National Institutes of Health, Bethesda, Maryland). Each 12-bit image was linearly scaled to 8 bits for processing, and was well within the dynamic range of the CCD. The areas of interest corresponding to 
each spot on the coverslip were extracted using a binary mask in the form of the photomask (Figure 2). The average fluorescence from each of the three circular areas in each row was computed using the quantitation tools in NIH Image, and the average of these three values was used as the initial signal from the region ( \pm standard deviation). The rectangular areas at the end of each row were used as a standardization signal by taking the average intensity of each rectangle and obtaining the norm of the two values. The signals from each of the circular regions were then expressed relative to the norm from the rectangular signals. Since the illumination sources for the images were directed parallel to the rows, and since the rows contained the areas of interest, the greatest need for correcting for variation in lighting intensity was in the horizontal direction.

\section{EXPERIMENTAL RESULTS}

A typical experiment for the detection of low concentrations of three Cy5-labeled antibodies, as well as the signal from a fourth labeled antibody used for correcting for uneven illumination is shown in Figure 3. In Figure 3(A), the Cy5-rabbit anti-horse IgG $(500 \mathrm{ng} / \mathrm{ml})$ binds to the rectangular regions at the edges of the pattern. The resulting fluorescence (after subtraction of a PBS background) indicates nonuniform lighting across the sample, and in particular, the central horizontal row of antibodies would be expected to be excited to a greater extent than those of either the top or bottom rows. Thus, for quantitation of samples, it is necessary to have such continuous controls in the array.

Figure 3(A) also indicates a degree of crossreactivity between the Cy5-rabbit anti-horse IgG antibody and goat IgG (lower row of circular spots). When examined separately, the other antibodies used do not show detectable cross-reactivity (data not shown). This background reactivity must consequently be subtracted from the specific signals detected.

The images in Figure 3 also show the sequential addition of $300 \mathrm{ng} / \mathrm{ml}$ samples of Cy5-labeled rabbit anti-goat IgG (panel B), rabbit anti-chicken IgG (panel C), and rabbit anti-mouse IgG (panel D). Even at these relatively low concentrations of antigen, excellent sensitivity was observed, and each signal could be easily discerned, with a signal difference of 10 to 30 standard deviations above background noise levels. Typically, the $50 \mathrm{ng} / \mathrm{ml}$ fluorescence intensities were 17 to $23 \%$ higher than the PBS background, and 6 to 8 standard deviations above background noise following PBS image subtraction. This assay could also detect mixtures of labeled rabbit anti-species solutions at concentrations of $100 \mathrm{ng} / \mathrm{ml}$ (data not shown), making it a simple and rapid device for detecting multiple analytes within a single $150-\mu 1$ sample.

Due to the linear response of CCD sensors, quantitation of analyte concentrations could also be per-
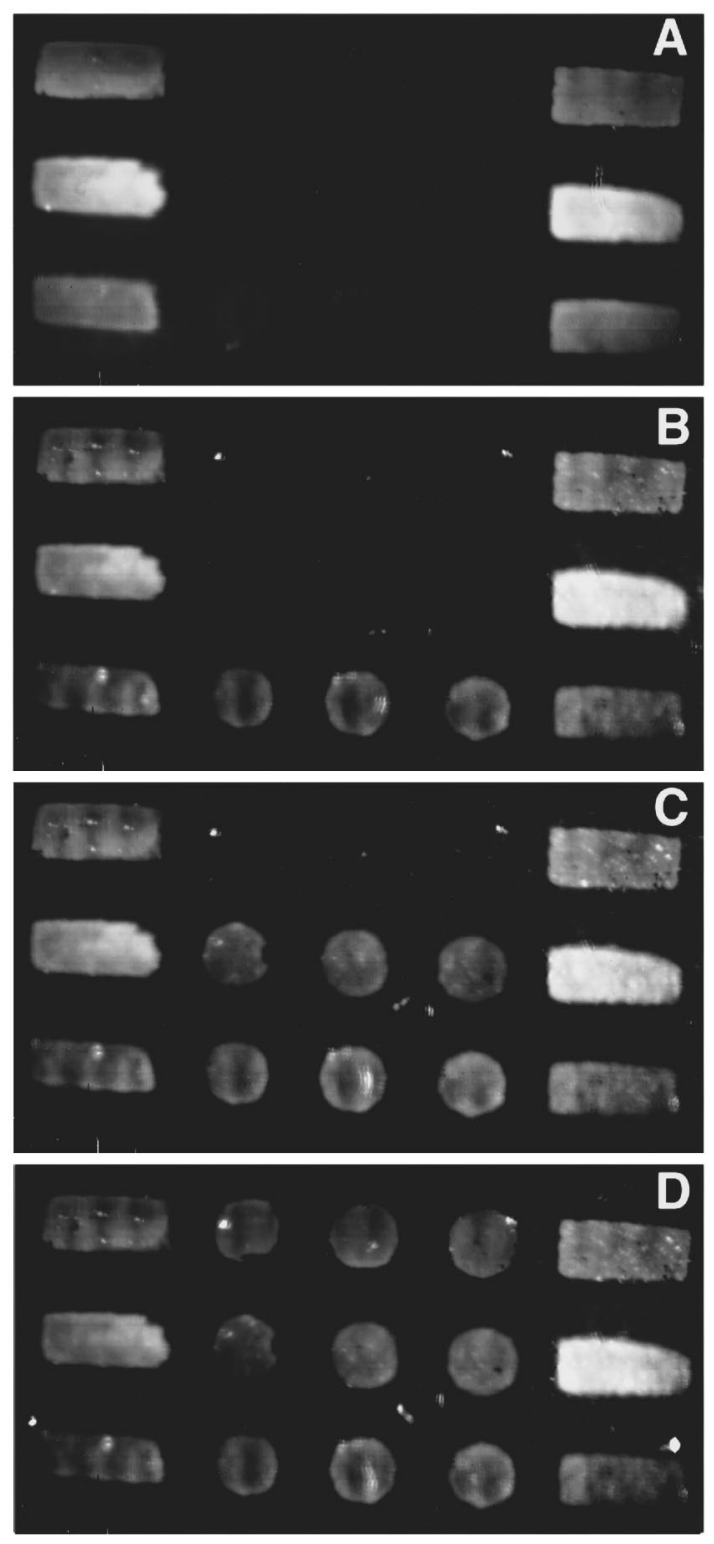

Fig. 3 Detection of multiple analytes using the biosensor. Horse $\lg G$ was immobilized in the rectangular regions of the pattern, while goat, chicken, and mouse lgG were immobilized in rows in circular areas. Images shown are from the CCD sensor after background subtraction of a PBS buffer image. Panels shown are for incubation of the slide with (A) $500 \mathrm{ng} / \mathrm{ml}$ Cy5-labeled rabbit antihorse $\lg G$, and $300 \mathrm{ng} / \mathrm{ml}$ concentrations of Cy5-labeled (B) rabbit anti-goat $\lg G,(C)$ rabbit anti-chicken $\lg G$, and (D) rabbit antimouse lgG. The circular regions are $15 \mathrm{~mm}$ in diameter, while the rectangular regions are $32 \times 14 \mathrm{~mm}$.

formed. Increasing concentrations of Cy5-labeled antibody were placed over the coverslip array and allowed to stand for $15 \mathrm{~min}$. After washing, the images due to fluorescence of Cy5 were collected, and the initial image captured prior to addition of the antibody was subtracted from the image for each concentration. The resulting subtracted images for $100 \mathrm{ng} / \mathrm{ml}$ solutions of the three Cy5-labeled rabbit anti-species are shown in Figure 4. As was indicated in Figure 3, the signals from the top and bot- 

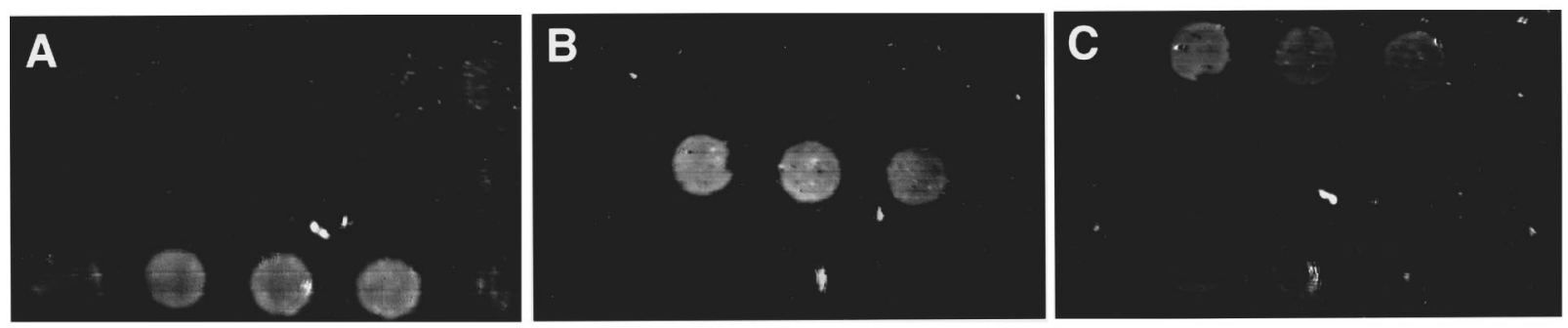

Fig. 4 Quantitation of detected fluorescence. The coverslip shown in Figure 3 was sequentially exposed to increasing concentrations of Cy5-labeled antibodies. After incubating with antibody, an image was taken and the image taken prior to addition of antibody was subtracted from it. This resulted in signals due solely to the antibody of interest. Shown are images resulting from $100 \mathrm{ng} / \mathrm{ml}$ of Cy5-labeled (A) rabbit anti-goat $\lg G$, (B) rabbit anti-chicken $\lg G$, and (C) rabbit anti-mouse $\lg G$.

tom rows are less intense than those in the middle. This is in part due to uneven illumination, but may also be the result of contributions from the varying avidities of the antibodies.

The average fluorescence signals from the indicated spots were quantitated with NIH Image for $25,50,100$, and $300 \mathrm{ng} / \mathrm{ml}(\sim 0.2$ to $1.9 \mathrm{nM})$ samples of antibody solutions. These values were then normalized to the average fluorescence from the rectangular regions of the same row in the image (we assumed a smooth gradation of lighting intensities across the rows). The normalized data are plotted in Figure 5 to show the concentration response by the sensor. For both Cy5-labeled rabbit anti-chicken IgG and rabbit antimouse IgG, the signal from the $25-\mathrm{ng} / \mathrm{ml}$ solution was below the detection limit of the system, while the $50-\mathrm{ng} / \mathrm{ml}$ signal was significantly above background. For the coverslip examined in Figures 3 and 4, even in the

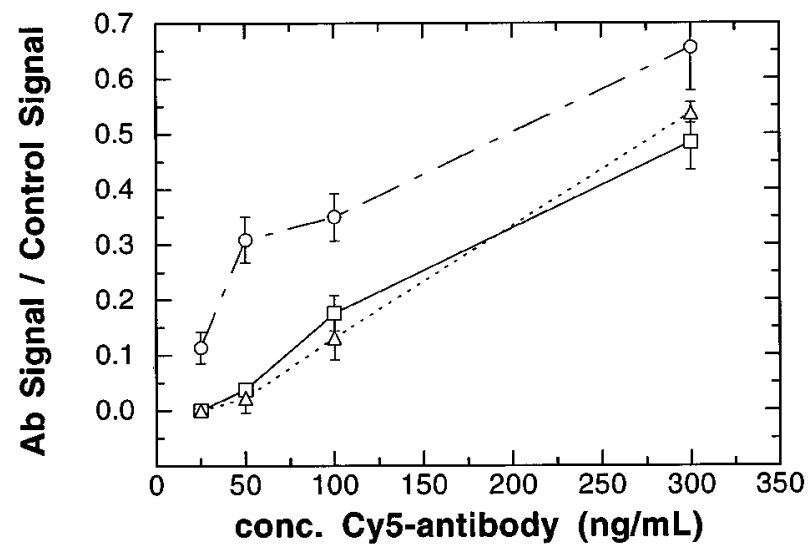

Fig. 5 Response of the biosensor to increasing concentration of Cy5-labeled antibody. The responses were determined from the subtracted images for each concentration as shown in Figure 4. The fluorescence intensities for each of the three circular regions was normalized to the average intensities from the rectangular regions for the row of interest shown in Figure 3(A). Data shown are for $(O)$ rabbit anti-goat $\lg G,(\square)$ rabbit anti-chicken $\lg G$, and $(\triangle)$ rabbit anti-mouse lgG. Data shown are from a representative assay and are the mean \pm standard deviation of three spots on the same coverslip. presence of background Cy5-rabbit anti-horse IgG signals, Cy5-labeled rabbit anti-goat IgG could be detected at $25 \mathrm{ng} / \mathrm{ml}$. However, in routine use of a series of coverslips $(n>5), 50 \mathrm{ng} / \mathrm{ml}$ was the minimal concentration that could be consistently detected with reliability in our device. Concentrations of $100 \mathrm{ng} / \mathrm{ml}$ and higher were easily detected for all antigens examined, as shown in Figures 3 through 5 .

\section{DiscUSSION}

In this report we present a new design for a multianalyte biosensor based on fluorescence detection and scientific grade CCD technology. We use antigens immobilized on glass coverslips to concentrate analyte within patterned wells, and can easily detect antibody concentrations as low as $50 \mathrm{ng} / \mathrm{ml}$ (0.29 $\mathrm{nM}$ for $\sim 170 \mathrm{kD}$ antibody analytes). This design can be used to construct multianalyte biosensors for many antigens, including biologically hazardous agents, environmental pollutants, explosives, or drugs of abuse. ${ }^{23-25}$ Further, in our laboratory we have used this design for performing sandwich immunoassays (data not shown) for analytes without endogenous fluorescence. It is also possible to use the device with competitive immunoassays to detect small molecules.

Our design has many advantages over currently used techniques, due to its high sensitivity and ease of use: (1) microscopes or sophisticated optics and camera systems are not necessary for interrogating the binding areas; (2) coverslips are disposable, and therefore many assays can be performed quickly; and (3) from the linearity of the CCD detector, quantitation of antigen concentrations is possible. Perhaps most important, the small footprint and low profile of the device and its ability to work at room temperature mean the design can be easily incorporated into portable or field biosensors for on-site detection of analytes. In this regard, due to the near-IR excitation and fluorescence of the $\mathrm{Cy} 5$ dyes, the lamp system used here for illumination can be replaced with inexpensive 635-nm diode lasers. Evanescent excitation rather than overhead il- 
lumination could then be used to construct a smaller device. Our preliminary results indicate that evanescent excitation by such a laser system provides results comparable to those reported here. Truly portable biosensors based on these systems are under development in our laboratory.

\section{Acknowledgments}

The authors wish to thank Sarah Golightly and Drs. George Anderson, David Conrad, and Jeff Ives for helpful insights. Dr. Wadkins was supported through a National Research Council/National Science Foundation Associateship at the Naval Research Laboratory. The views expressed in this work are those of the authors, and do not necessarily represent those of the U.S. government, the Department of Defense, or the U.S. Navy.

\section{REFERENCES}

1. D. L. Wise and L. B. Wingard, Jr. (Eds.), Biosensor with Fiberoptics, Humana Press, Clifton, NJ (1991).

2. A. E. G. Cass, Biosensors, Oxford Univ. Press, New York (1990).

3. O. S. Wolfbeis (Ed.), Fiber Optic Chemical Sensors and Biosensors, Vols. I and II, CRC Press, Boca Raton, FL (1991).

4. D. Christensen, T. Johannson, D. Petelenz, "Biosensor development at the University of Utah," IEEE Engineer. Med. Biol. 13, 388-395 (1994).

5. R. B. Thompson, and F. S. Ligler, "Chemistry and technology of evanescent wave biosensors," in Biosensors with Fiberoptics, L. B. Wingard and D. L. Wise, Eds., pp. 111-138, Humana Press, NJ (1991).

6. S. Y. Rabbany, B. L. Donner, and F. S. Ligler, "Optical immunosensors," Crit. Rev. Biomed. Eng. 22, 307-346 (1994).

7. J. Kimura, Y. Kawana, and T. Kuriyama, "An immobilized enzyme membrane fabrication method using an ink jet nozzle," Biosensors 4, 41-52 (1988).

8. Y. Hanazato, M. Nakako, S. Shiono, and M. Maeda, "Integrated multi-biosensors based on an ion-selective fieldeffect transistor using photolithographic techniques," IEEE Trans. Electron Devices 36(7), 1303-1309 (1989).

9. K. S. Bronk, and D. R. Walt, "Fabrication of patterned sensor arrays with aryl azides on a polymer-coated imaging optical fiber bundle," Anal. Chem. 66(20), 3519-3520 (1994).

10. A. C. Pease, D. Solas, E. J. Sullivan, M. T. Cronin, C. P. Holmes, and S. P. A. Fodor, "Light-generated oligonucleotide arrays for rapid DNA sequence analysis," Proc. Natl. Acad. Sci. USA 91, 5022-5026 (1994).

11. M. Eggers, M. Hogan, R. K. Reich, J. Lamture, D. Ehrlich, M. Hollis, B. Kosicki, T. Powdrill, K. Beattie, S. Smith, R. Varma, R. Gangadharan, A. Mallik, B. Burke, and D. Wallace, "A microchip for quantitative detection of molecules utilizing luminescent and radioisotope reporter groups," BioTechniques 17(3), 516-524 (1994).
12. S. P. A. Fodor, J. L. Read, M. C. Pirrung, L. Stryer, A. T. Lu, and D. Solas, "Light-directed, spatially addressable parallel chemical synthesis," Science 251, 767-773 (1991).

13. S. Britland, E. Perez-Arnaud, P. Clark, B. McGinn, P. Connolly, and G. Moores, "Micropatterning proteins and synthetic peptides on solid supports: a novel application for microelectronics fabrication technology," Biotechnol. Prog. 8, 155-160 (1992).

14. Z. Guo, R. A. Guilfoyle, A. J. Thiel, R. Wang, and L. M. Smith, "Direct fluorescence analysis of genetic polymorphisms by hybridization with oligonucleotide arrays on glass supports," Nucleic Acids Res. 22(24), 5456-5465 (1994).

15. D. J. Pritchard, H. Morgan, and J. M. Cooper, "Patterning and regeneration of surfaces with antibodies," Anal. Chem. 67(19), 3605-3607 (1995).

16. Y. Zhou, J. V. Magill, W. Cushley, P. J. R. Laybourn, and R. M. De La Rue, "Multichannel evanescent fluorescence immunosensing using potassium and sodium ion-exchanged patterned waveguides," J. Mol. Electronics 7, 135-149 (1991).

17. Y. Zhou, J. V. Magill, R. M. De La Rue, and P. J. R. Laybourn, "Evanescent fluorescence immunoassays performed with a disposable ion-exchanged patterned waveguides," Sensors Actuators B 11, 245-250 (1993).

18. V. Hlady, J. N. Lin, and J. D. Andrade, "Spatially resolved detection of antibody-antigen reaction on solid/liquid interface using total internal reflection excited antigen fluorescence and charge-coupled device detection," Biosensors Bioelectronics 5, 291-301 (1990).

19. P. L. Southwick, L. A. Ernst, E. W. Tauriello, S. R. Parker, R. B. Mujumdar, S. R. Mujumdar, H. A. Clever, and A. S. Waggoner, "Cyanine dye labeling reagents-carboxy-methylindocyanine succinimidyl esters," Cytometry 11, 418-430 (1990).

20. R. B. Mujumdar, L. A. Ernst, S. R. Mujumdar, C. J. Lewis, and A. S. Waggoner, "Cyanine dye labeling reagents: sulfoindocyanine succinimidyl esters," Bioconjugate Chem. 4, 105-111 (1993).

21. B. Burke and S. Gajar, "Dynamic suppression of interfacestate dark current in buried channel CCDs," IEEE Trans. Electron Devices 38 (2), 285-290 (1991).

22. S. K. Bhatia, L. C. Shriver-Lake, K. J. Prior, J. H. Georger, J. M. Calvert, R. Bredehorst, and F. S. Ligler, "Use of Thiolterminal silanes and heterobifunctional crosslinkers for immobilization of antibodies on silica surfaces," Anal. Biochem. 178, 408-413 (1989).

23. F. S. Ligler, J. P. Golden, L. C. Shriver-Lake, R. A. Ogert, D. Wijesuria, and G. P. Anderson, "Fiber-optic biosensor for the detection of hazardous materials," Immunomethods $\mathbf{3}$, 122-127 (1993).

24. L. C. Shriver-Lake, R. A. Ogert, and F. S. Ligler, "A fiberoptic evanescent-wave immunosensor for large molecules," Sensors Actuators B 11, 239-243 (1993).

25. L. C. Shriver-Lake, K. A. Breslin, P. T. Charles, D. W. Con$\mathrm{rad}$, J. P. Golden, and F. S. Ligler, "Detection of TNT in water using an evanescent wave fiber-optic biosensor," Anal. Chem. 34, 2431-2435 (1995). 